\title{
Non-universal gaugino mass GUT models in the light of dark matter and LHC constraints
}

\author{
Joydeep Chakrabortty, ${ }^{a}$ Subhendra Mohanty ${ }^{b}$ and Soumya Rao ${ }^{c}$ \\ ${ }^{a}$ Department of Physics, Indian Institute of Technology, \\ Kanpur-208016, India \\ ${ }^{b}$ Physical Research Laboratory, \\ Ahmedabad-380009, India \\ ${ }^{c}$ Department of Theoretical Physics and Centre for Theoretical Sciences, \\ Indian Association for the Cultivation of Science, \\ 2A 8 2B Raja S.C. Mullick Road, Kolkata-700032, India \\ E-mail: joydeep@iitk.ac.in, mohanty@prl.res.in, tpsr2@iacs.res.in
}

ABSTRACT: We perform a comprehensive study of $\mathrm{SU}(5), \mathrm{SO}(10)$ and $\mathrm{E}(6)$ supersymmetric GUT models where the gaugino masses are generated through the F-term breaking vacuum expectation values of the non-singlet scalar fields. In these models the gauginos are nonuniversal at the GUT scale unlike in the mSUGRA scenario. We discuss the properties of the LSP which is stable and a viable candidate for cold dark matter. We look for the GUT scale parameter space that leads to the the lightest SM like Higgs mass in the range of 122-127 GeV compatible with the observations at ATLAS and CMS, the relic density in the allowed range of WMAP-PLANCK and compatible with other constraints from colliders and direct detection experiments. We scan universal scalar $\left(m_{0}^{G}\right)$, trilinear coupling $A_{0}$ and $\mathrm{SU}(3)_{C}$ gaugino mass $\left(M_{3}^{G}\right)$ as the independent free parameters for these models. Based on the gaugino mass ratios at the GUT scale, we classify 25 SUSY GUT models and find that of these only 13 models satisfy the dark matter and collider constraints. Out of these 13 models there is only one model where there is a sizeable SUSY contribution to muon $(g-2)$.

KeYwords: Supersymmetry Phenomenology

ARXiv EPRINT: 1310.3620 


\section{Contents}

1 Introduction 1

2 SUSY GUT and non-universal gauginos 2

3 Results 4

3.1 Relic density and collider constraints 4

3.2 Direct detection constraints 11

4 Muon $(g-2) \quad 13$

5 Conclusions $\quad 15$

\section{Introduction}

Supersymmetry (SUSY) is an aesthetically appealing model which provides a natural mechanism to stabilise the Higgs mass and solves the gauge hierarchy problem of the Standard Model. The general Supersymmetric Standard Model at the electroweak scale has more than a hundred parameters which make the predictability of such models questionable. An economical Supersymmetric Standard Model can be constructed which contains only a few free parameters known as the constrained Minimal Supersymmetric Standard Model (cMSSM), which relates to the high scale minimal supergravity models (mSUGRA) through renormalisation groups. In mSUGRA there are only 5 parameters: universal scalar mass $m_{0}$, universal gaugino mass $M_{1 / 2}$, $\tan \beta$, sign of $\mu(\operatorname{sgn}(\mu))$ and universal tri-linear couplings $A_{0}$. The lightest supersymmetric particle (LSP) is mostly bino-like. But the recent LHC data is ruling out most of its parameter space for obtaining the WMAP-PLANCK measured relic density of bino as cold dark matter. But it is not necessary to have all the gauginos unified at the unification scale.

It has been noted in [1-7] that in Supersymmetric Grand Unified Theories (SUSY GUTs) the boundary conditions at the high scale itself can be different than that in mSUGRA. The gaugino masses can be non-universal at the GUT scale itself. The renormalisation group evolutions (RGEs) further change their ratios at the electroweak scale and thus the phenomenology of such models can be completely different compared to mSUGRA. But these non-universalities in SUSY GUT models are completely determined from the group theoretic structure of the symmetry breaking scalar fields. In [1-7] these non-universal gaugino mass ratios were first calculated for SU(5) group with 24-, 75-, and 200- dimensional scalar fields. Later in [8-11] the non-universal gaugino mass ratios are presented for all possible breaking patterns having all possible scalar fields for $\mathrm{SO}(10)$, and E(6) GUT gauge groups. 
These non-universal models are clear departure from mSUGRA in the boundary conditions. Thus different non-universalities lead to different kind of LSP scenarios. Some recent papers has partly grabbed the impact of non-universality feature in either minimal or nonminimal version of models in the context of dark matter search, see for example [10, 12-30].

In this paper we have encapsulated the parameter space for all models (25) arising from different GUT gauge groups, like $\mathrm{SU}(5), \mathrm{SO}(10)$, and $\mathrm{E}(6)$ and the symmetry breaking patterns from all the possible scalar representations which can break the F-term and gauge symmetries as well. These give rise to different mass ratios of the three gauginos at the GUT scale. Here we have considered only those models for which all of them are non-zero at the unification scale.

Running the masses down to the electroweak scale we get the ratios $M_{1}: M_{2}: M_{3}$ for different models which are quite distinct from the mSUGRA relation $1: 2: 6.7$ at electroweak scale. Here $M_{1}, M_{2}, M_{3},{ }^{1}$ are the gaugino masses corresponding to the $\mathrm{U}(1)_{Y}, \mathrm{SU}(2)_{L}, \mathrm{SU}(3)_{C}$ gauge groups respectively. We scan the parameters $M_{3}^{G}, m_{0}^{G}, A_{0}$, $\tan \beta$ and test the range of parameters for each model which give the lightest Higgs mass in the range $122 \mathrm{GeV}<M_{h}<127 \mathrm{GeV}[31,32]$, and the dark matter relic density within 3 -sigma of the WMAP-PLANCK $[33,34]$ measured band $0.112<\Omega h^{2}<0.128$. In addition we have other constraints: within the allowed parameter space the contribution to the $B_{s} \rightarrow X_{s} \gamma$ [35], $B_{s} \rightarrow \mu^{+} \mu^{-}$[36] and the muon $(g-2)$ [37-39] must satisfy the experimental bounds. We have also set the lower limit on the gluino mass $\left(m_{\tilde{g}}\right)$ to be $1.4 \mathrm{TeV} .^{2}$ Once these criterion are satisfied we compute the best fit value for the SUSY contribution to muon $(g-2)$ within the parameter space of the models constrained by the other experimental limits.

Of the 25 models examined we find that only 13 models satisfy the collider and dark matter experimental constraints and we find however that none of these 13 models explain the experimental value of muon $(g-2)$ [37-39]. The other 12 models are mainly ruled out when we impose light Higgs mass and 3-sigma relic density constraints together. The largest contribution to muon $(g-2)$ comes from the the models where the gaugino mass ratio at GUT scale is $M_{1}: M_{2}: M_{3} \equiv-1 / 2:-3 / 2: 1$ and this model has a bino like dark matter with mass $177 \mathrm{GeV}$.

There are five wino, five bino and three higssino dark matter models which give the WMAP-PLANCK relic density. Some of the models can be probed by the XENON1T [40] and Super-CDMS [41] experiments and one model is ruled out by XENON100 [42].

\section{SUSY GUT and non-universal gauginos}

Supersymmetry and Grand Unified Theory both have different motivations to be suitable theories beyond the Standard Model. Supersymmetry justifies the gauge hierarchy problem and predicts many other superpartners of SM particles. In R-parity conserving SUSY theories LSP is stable and can be a viable cold dark matter candidate. Here we will focus

\footnotetext{
${ }^{1}$ We define the GUT scale input of these parameters as $M_{i}^{G}$.

${ }^{2}$ Though it is not playing any crucial role in our analysis as within the parameter space allowed by the other constraints $m_{\tilde{g}}$ is more than $1.8 \mathrm{TeV}$ or so.
} 
only on the neutralino LSPs. Within this framework SUSY is expected to explain the observed relics of the Universe. Added with these nice outcomes the extra feature of this theory in the GUT framework is very encouraging. SUSY improves the gauge coupling unification in most of the GUT models. Thus SUSY GUT models are phenomenologically interesting and motivating.

The GUT symmetry is broken when a non-singlet direction under that gauge group acquires vacuum expectation value. In SUSY GUT unified frame work most of the couplings (masses) are degenerate at the unification scale. In its minimal form all the gauginos and scalars are universal respectively. The other free parameters are tri-linear coupling $\left(A_{0}\right)$ which is also universal, $\tan \beta$ (ratio of the vacuum expectation values, vev, of two Higgs doublets), and sign of $\mu$ (Higgs parameter). But we can have other possibilities, like gauginos or scalars are non-universal at the High scale themselves when we work under SUSY GUT framework. The scalars that cause the GUT symmetry breaking may develop a F-term breaking vev. Thus GUT and supersymmetry are broken via a single scalar but through the vevs in different directions. The gauge kinetic term can be recast in a much simpler form as: $\frac{\eta}{M} \operatorname{Tr}\left(F_{\mu \nu} \Phi F^{\mu \nu}\right)$ where $\eta$ is dimensionless parameter, $M=M_{\mathrm{Pl}} / \sqrt{8 \pi}$ (reduced Planck mass). As $F_{\mu \nu}$ transforms as adjoint of the unbroken GUT groups, $\Phi$ belongs to the symmetric product of the two adjoints.

In this paper we have worked on $\mathrm{SU}(5), \mathrm{SO}(10), \mathrm{E}(6)$ GUT groups, thus the choices of scalars are as following:

$$
\begin{aligned}
\mathrm{SU}(5) & \Rightarrow(24 \otimes 24)_{\mathrm{sym}}=1 \oplus 24 \oplus 75 \oplus 200, \\
\mathrm{SO}(10) & \Rightarrow(45 \otimes 45)_{\mathrm{sym}}=1 \oplus 54 \oplus 210 \oplus 770, \\
\mathrm{E}(6) & \Rightarrow(78 \otimes 78)_{\mathrm{sym}}=1 \oplus 650 \oplus 2430,
\end{aligned}
$$

where $24,45,78$ are the dimensions of the adjoint representations of $\mathrm{SU}(5), \mathrm{SO}(10), \mathrm{E}(6)$ respectively.

It has been noted earlier that these operators also change the gauge coupling unification conditions at the high scale and in many cases it improves the unifications, see for example [43-46]. As these scalars are non-singlet, their vev treat the SM gauginos in different footing. Thus the SM gauge fields, i.e. the gauge couplings are scaled differently. These types of operators can inject non-universality in the gaugino masses.

In $\mathrm{SU}(5)$ models with only possible breaking pattern: $\mathrm{SU}(5) \rightarrow \mathrm{SU}(3) \otimes \mathrm{SU}(2) \otimes \mathrm{U}(1)$ the scalar fields of 24,75 and 200 dimensions lead to three different set of non-universal gaugino mass ratios. But as the ranks of $\mathrm{SO}(10)$ and $\mathrm{E}(6)$ are larger than that of the SM there are more than one possible breaking patterns of these GUT symmetry groups. We have noted the gaugino mass ratios for the following intermediate breaking patterns of $\mathrm{SO}(10): \mathrm{SU}(5) \otimes \mathrm{U}(1), \mathrm{SU}(4) \otimes \mathrm{SU}(2) \otimes \mathrm{SU}(2)$, and for $\mathrm{E}(6)$ we have considered $\mathrm{SO}(10)^{\prime} \otimes$ $\mathrm{U}(1), \mathrm{SU}(3) \otimes \mathrm{SU}(3) \otimes \mathrm{SU}(3), \mathrm{SU}(6) \otimes \mathrm{SU}(2)$. Though the group theoretic structures are similar in few cases but as the SM symmetry is realised in different ways the non-universal gaugino mass ratios are different for those models. For example $\mathrm{SU}(5) \otimes \mathrm{U}(1)$ is a maximal subgroup of $\mathrm{SO}(10)$. In normal $\mathrm{SU}(5)$ model the extra $\mathrm{U}(1)$ does not contribute in $\mathrm{U}(1)_{Y}$ of SM, but in flipped SU(5) model the hypercharge generator of SM is a linear combination 


\begin{tabular}{|c|c|c|c|}
\hline Model Number & $\begin{array}{c}M_{1}: M_{2}: M_{3} \\
\left(\text { at } \mathrm{M}_{X}\right)\end{array}$ & $\begin{array}{c}M_{1}: M_{2}: M_{3} \\
\left(\text { at } \mathrm{M}_{\mathrm{EW}}\right)\end{array}$ & Model \\
\hline \hline 1 & $-19 / 5: 1: 1$ & $-19 / 5: 2: 6$ & $\mathrm{SO}(10) \stackrel{(1,0) \subset 210}{\longrightarrow}(\mathrm{SU}(5) \otimes \mathrm{U}(1))_{\text {flipped }}$ \\
\hline 2 & $-3: 1: 1$ & $-3: 2: 6$ & $\mathrm{E}(6) \stackrel{(189,1) \subset 2430,650}{\longrightarrow}\left(\mathrm{SU}(6) \otimes \mathrm{SU}(2)_{X}\right)$ \\
\hline 3 & $-13 / 5: 1: 1$ & $-13 / 5: 2: 6$ & $\mathrm{E}(6) \stackrel{(1,1) \subset 650}{\longrightarrow}\left(\mathrm{SU}(6) \otimes \mathrm{SU}(2)_{R}\right)$ \\
\hline 4 & $-22 / 5: 1: 1$ & $-22 / 5: 2: 6$ & $\mathrm{E}(6) \stackrel{(1,0) \subset 650}{\longrightarrow}(\mathrm{SO}(10) \otimes \mathrm{U}(1))_{\text {flipped }}$ \\
\hline 5 & $41 / 15: 1: 1$ & $41 / 15: 2: 6$ & $\mathrm{E}(6) \stackrel{(1,1) \subset 2430}{\longrightarrow}\left(\mathrm{SU}(6) \otimes \mathrm{SU}(2)_{R}\right)$ \\
\hline 6 & $122 / 5: 1: 1$ & $122 / 5: 2: 6$ & $\mathrm{E}(6) \stackrel{(1,0) \subset 2430}{\longrightarrow}(\mathrm{SO}(10) \otimes \mathrm{U}(1))_{\text {flipped }}$ \\
\hline 7 & $-101 / 10:-3 / 2: 1$ & $-101 / 10:-3: 6$ & $\mathrm{SO}(10) \stackrel{(24,0) \subset 770}{\longrightarrow}(\mathrm{SU}(5) \otimes \mathrm{U}(1))_{\text {flipped }}$ \\
\hline 8 & $77 / 5: 1: 1$ & $77 / 5: 2: 6$ & $\mathrm{SO}(10) \stackrel{(1,0) \subset 770}{\longrightarrow}(\mathrm{SU}(5) \otimes \mathrm{U}(1))_{\text {flipped }}$ \\
\hline 9 & $10: 2: 1$ & $10: 4: 6$ & $\mathrm{SO}(10) \stackrel{(200) \subset 770}{\longrightarrow} \mathrm{SU}(5)$ \\
\hline
\end{tabular}

Table 1. Ratios of gaugino masses that lead to $M_{1}>M_{2}$ at $\operatorname{EWSB}\left(M_{\mathrm{EW}}\right)$ Scale.

of this $\mathrm{U}(1)$ and another Abelian group coming from $\mathrm{SU}(5)$. In these two cases the ratio of the gaugino masses at the GUT scale are different from each other. Here we have tabulated 24 different types of non-universal gaugino mass ratios discarding the possibility of one of the gauginos has zero mass at the high scale. It is very interesting to note that unlike the mSUGRA scenario here we can have either $M_{1}>M_{2}$ or $M_{1}<M_{2}$ and even $M_{1} \simeq M_{2}$ at the electroweak scale. Thus where in mSUGRA we have mostly bino-like Lightest Supersymmetric Particle (LSP), in these SUSY-GUT frame work because of the non-universality one can have purely bino- or wino- or higgsino- dominated LSP or a mixed one also.

Here we briefly mention our model identifications depending on the GUT groups, choices of scalar fields and symmetry breaking patterns, see tables 1 and 2 . Here we would like to pass a remark that while calculating these gaugino mass ratios for different models it has been assumed that all the intermediate symmetry scales are same as the unification (GUT) scale, i.e., the GUT symmetry is broken to the SM gauge group at the unification scale itself.

\section{Results}

We examine the different non-universal gaugino mass models in the light of relic density, direct detections and collider bounds. We have classified all the models in three categories depending on the compositions of the LSPs: bino-dominated, wino-dominated, and higgsino-dominated.

\subsection{Relic density and collider constraints}

We have used the following constraints in our analysis and determine which of the 25 models arising from non-singlet Higgs pass these tests: 


\begin{tabular}{|c|c|c|c|}
\hline Model Number & $\begin{array}{c}M_{1}: M_{2}: M_{3} \\
\quad\left(\text { at } \mathrm{M}_{X}\right)\end{array}$ & $\begin{array}{c}M_{1}: M_{2}: M_{3} \\
\quad\left(\text { at } \mathrm{M}_{\mathrm{EW}}\right)\end{array}$ & Model \\
\hline 10 & $\frac{9}{5}: 1: 1$ & $\frac{9}{5}: 2: 6$ & $\mathrm{E}(6) \stackrel{(405,1) \subset 2430}{\longrightarrow}\left(\mathrm{SU}(6) \otimes \mathrm{SU}(2)_{R}\right)$ \\
\hline 11 & $-5: 3: 1$ & $-5: 6: 6$ & $\mathrm{SO}(10) \stackrel{(75) \subset 770}{\longrightarrow} \mathrm{SU}(5)$ \\
\hline 12 & $1: 35 / 9: 1$ & $1: 70 / 9: 6$ & $\mathrm{E}(6) \stackrel{(1,1) \subset 2430}{\longrightarrow}\left(\mathrm{SU}(6) \otimes \mathrm{SU}(2)_{L}\right)$ \\
\hline 13 & $1:-5: 1$ & $1:-10: 6$ & $\mathrm{E}(6) \stackrel{(1,1) \subset 650}{\longrightarrow}\left(\mathrm{SU}(6) \otimes \mathrm{SU}(2)_{L}\right)$ \\
\hline 14 & $-3 / 5: 1: 1$ & $-3 / 5: 2: 6$ & $\mathrm{E}(6) \stackrel{(189,1) \subset 650,2430}{\longrightarrow}\left(\mathrm{SU}(6) \otimes \mathrm{SU}(2)_{R}\right)$ \\
\hline 15 & $-1 / 5:-1: 1$ & $-1 / 5:-2: 6$ & $\mathrm{E}(6) \stackrel{(35,1) \subset 650}{\longrightarrow}\left(\mathrm{SU}(6) \otimes \mathrm{SU}(2)_{R}\right)$ \\
\hline 16 & $1 / 10: 5 / 2: 1$ & $1 / 10: 5: 6$ & $\mathrm{E}(6) \stackrel{(770,0) \subset 2430}{\longrightarrow}(\mathrm{SO}(10) \otimes \mathrm{U}(1))_{\text {flipped }}$ \\
\hline 17 & $1 / 10:-3 / 2: 1$ & $1 / 10:-3: 6$ & $\mathrm{E}(6) \stackrel{(54,0) \subset 650}{\longrightarrow}(\mathrm{SO}(10) \otimes \mathrm{U}(1))_{\text {flipped }}$ \\
\hline 18 & $2 / 5: 2: 1$ & $2 / 5: 4: 6$ & $\begin{array}{l}\mathrm{E}(6) \stackrel{(770,0) \subset 2430}{\longrightarrow}(\mathrm{SO}(10) \otimes \mathrm{U}(1))_{\text {flipped }} \\
(\mathrm{SO}(10) \otimes \mathrm{U}(1))_{\text {flipped }} \stackrel{(200) \subset 770}{\longrightarrow} \mathrm{SU}(5)\end{array}$ \\
\hline 19 & $-1 / 5: 3: 1$ & $-1 / 5: 6: 6$ & $\begin{array}{c}\mathrm{E}(6) \stackrel{(210,0),(770,0) \subset 650,2430}{\longrightarrow}(\mathrm{SO}(10) \otimes \mathrm{U}(1))_{\text {flipped }} \\
\quad(\mathrm{SO}(10) \otimes \mathrm{U}(1))_{\text {flipped }} \stackrel{(75) \subset 210,770}{\longrightarrow} \mathrm{SU}(5)\end{array}$ \\
\hline 20 & $5 / 2:-3 / 2: 1$ & $5 / 2:-3: 6$ & $\begin{array}{l}\mathrm{E}(6) \stackrel{(770,0) \subset 2430}{\longrightarrow}(\mathrm{SO}(10) \otimes \mathrm{U}(1))_{\text {flipped }} \\
(\mathrm{SO}(10) \otimes \mathrm{U}(1))_{\text {flipped }} \stackrel{(24) \subset 770}{\longrightarrow} \mathrm{SU}(5)\end{array}$ \\
\hline 21 & $-1 / 5:-3 / 2: 1$ & $-1 / 5:-3: 6$ & $\begin{array}{l}\mathrm{E}(6) \stackrel{(210,0) \subset 650,2430}{\longrightarrow}(\mathrm{SO}(10) \otimes \mathrm{U}(1))_{\text {flipped }} \\
\quad(\mathrm{SO}(10) \otimes \mathrm{U}(1))_{\text {flipped }} \stackrel{(24) \subset 210}{\longrightarrow} \mathrm{SU}(5)\end{array}$ \\
\hline 22 & $-1 / 5: 1: 1$ & $-1 / 5: 2: 6$ & $\begin{array}{l}\mathrm{E}(6) \stackrel{(210,0) \subset 650,2430}{\longrightarrow}(\mathrm{SO}(10) \otimes \mathrm{U}(1))_{\text {flipped }} \\
\quad(\mathrm{SO}(10) \otimes \mathrm{U}(1))_{\text {flipped }} \stackrel{(1) \subset 210}{\longrightarrow} \mathrm{SU}(5)\end{array}$ \\
\hline 23 & $19 / 10: 5 / 2: 1$ & $19 / 10: 5: 6$ & $\mathrm{SO}(10) \stackrel{(1,1) \subset 770}{\longrightarrow}\left(\mathrm{SU}(4) \otimes \mathrm{SU}(2)_{R}\right.$ \\
\hline 24 & $-1 / 2:-3 / 2: 1$ & $-1 / 2:-3: 6$ & $\begin{array}{c}\mathrm{SO}(10) \stackrel{(24) \subset 54,210,770}{\longrightarrow} \mathrm{SU}(5) \\
\mathrm{SO}(10) \stackrel{(24,0) \subset 54}{\longrightarrow}(\mathrm{SU}(5) \otimes \mathrm{U}(1))_{\text {flipped }} \\
\mathrm{SO}(10) \stackrel{(1,1) \subset 54}{\longrightarrow}\left(\mathrm{SU}(4) \otimes \mathrm{SU}(2)_{R}\right)\end{array}$ \\
\hline 25 & $7 / 10:-3 / 2: 1$ & $7 / 10:-3: 6$ & $\mathrm{SO}(10) \stackrel{(24,0) \subset 210}{\longrightarrow}(\mathrm{SU}(5) \otimes \mathrm{U}(1))_{\text {flipped }}$ \\
\hline
\end{tabular}

Table 2. Ratios of gaugino masses that lead to $M_{1}<M_{2}$ at $\operatorname{EWSB}\left(M_{\mathrm{EW}}\right)$ Scale.

1. Higgs mass bound from LHC [31, 32]

$$
122 \mathrm{GeV}<M_{h}<127 \mathrm{GeV}
$$

2. Relic density constraint from WMAP-PLANCK data at $3 \sigma[33,34]$

$$
0.1118<\Omega \mathrm{h}^{2}<0.1280
$$

3. Gluino mass $\left(m_{\tilde{g}}\right)>1.4 \mathrm{TeV}$. 
4. Branching fraction for $B_{s} \rightarrow X_{s} \gamma$ at $2 \sigma$ [35]

$$
3.05 \times 10^{-4}<\operatorname{BR}\left(B_{s} \rightarrow X_{s} \gamma\right)<4.05 \times 10^{-4}
$$

5. Branching fraction for $B_{s} \rightarrow \mu^{+} \mu^{-}$at $2 \sigma[47]$

$$
0.8 \times 10^{-4}<\mathrm{BR}\left(B_{s} \rightarrow \mu^{+} \mu^{-}\right)<6.2 \times 10^{-4}
$$

6. Ratio of branching fraction for $B_{u} \rightarrow \tau \nu_{\tau}$ in MSSM to that in SM at $3 \sigma[48,49]$

$$
0.46<\frac{\operatorname{BR}\left(B_{u} \rightarrow \tau \nu_{\tau}\right)_{\mathrm{MSSM}}}{\operatorname{BR}\left(B_{u} \rightarrow \tau \nu_{\tau}\right)_{\mathrm{SM}}}<1.78
$$

7. There is a discrepancy in anomalous muon magnetic moment, $a_{\mu} \equiv(g-2) / 2$, between experimental value [37-39] and SM prediction [50],

$$
\Delta a_{\mu}=a_{\mu}^{\exp }-a_{\mu}^{\mathrm{SM}}=(26.1 \pm 8.0) \times 10^{-10}
$$

We compute the SUSY contribution to $a_{\mu}$ for each of the models which satisfies the other criterion listed above. We find only one model where there is a substantial SUSY contribution with $a_{\mu}^{\mathrm{SUSY}}=2.65 \times 10^{-10}$.

For our analysis we use the two-loop RGE code SuSpect [51] to obtain the weak scale SUSY particle spectrum. In addition we use the MicrOMEGAs code [52] to evaluate low energy constraints like $B_{s} \rightarrow \mu^{+} \mu^{-}, B_{s} \rightarrow X_{s} \gamma$, muon $(g-2)$ and relic density. The parameter scan performed in this analysis takes the following ranges of parameters:

$$
\begin{aligned}
m_{0} & \in[100,2000] \mathrm{GeV}, \\
M_{3}^{G} & \in[800,2000] \mathrm{GeV}, \\
\operatorname{sgn}(\mu) & \equiv+,-.
\end{aligned}
$$

Here we define $M_{3}$ as $M_{3}^{G}$ at GUT scale and other gaugino masses $M_{1}, M_{2}$ are set by the gaugino mass ratios at that scale. We have performed our analysis for three different choices of tri-linear coupling $A_{0}=-1,0,1 \mathrm{TeV}$. We have chosen $\tan \beta=10$ unless mentioned otherwise.

We see that for large $A_{0}$ the $\tilde{\tau}$ mass becomes very large thereby precluding the staucoannihilation channel and as a result the relic density which depends on the stau coannihilation becomes too large (this holds for light bino DM and applies to model 24 only). Also very large $\tan \beta$ leads to conflict with the $B_{s} \rightarrow \mu^{+} \mu^{-}$constraint since the SUSY contribution to this process goes as $O\left(\tan ^{6} \beta\right)$.

In table 2 , model 24 which has a gaugino mass ratio of $-1 / 2:-3 / 2: 1$ having a bino LSP at low scale, is compatible with all the low energy constraints considered in this work. But it is mainly dependent on the stau coannihilation channel for achieving the correct relic density which means that one has to choose $m_{0}$ such that $\tilde{\tau}$ mass is quasi degenerate with the LSP mass. The sign of $\mu$ is chosen to be negative as that gives the a positive contribution to $(g-2)$. 


\begin{tabular}{|c|c|c|c|c|c|c|}
\hline Model no. & $M_{1}^{G}: M_{2}^{G}: M_{3}^{G}$ & $m_{0}(\mathrm{GeV})$ & $M_{3}^{G}(\mathrm{GeV})$ & $A_{0}(\mathrm{TeV})$ & $\tan \beta$ & $\operatorname{sgn}(\mu)$ \\
\hline 1 & $-\frac{19}{5}: 1: 1$ & 182 & 2038 & -1 & 10 & + \\
\hline 2 & $-3: 1: 1$ & 100 & 1620 & -1 & 10 & + \\
\hline 3 & $-\frac{13}{5}: 1: 1$ & 300 & 1320 & -1 & 10 & + \\
\hline 4 & $-\frac{22}{5}: 1: 1$ & 130 & 2055 & -1 & 10 & + \\
\hline 5 & $\frac{41}{15}: 1: 1$ & 300 & 1460 & -1 & 10 & + \\
\hline 9 & $10: 2: 1$ & 116 & 966 & -1 & 10 & + \\
\hline 10 & $\frac{9}{5}: 1: 1$ & 1000 & 1190 & -1 & 10 & + \\
\hline 11 & $-\frac{1}{5}: 3: 1$ & 2000 & 1650 & -4 & 40 & + \\
\hline 18 & $\frac{2}{5}: 2: 1$ & 200 & 1119 & -1 & 10 & + \\
\hline 19 & $-5: 3: 1$ & 789 & 1719 & -3.5 & 10 & + \\
\hline 20 & $\frac{5}{2}:-\frac{3}{2}: 1$ & 1900 & 1740 & -1 & 10 & - \\
\hline 22 & $-\frac{1}{5}: 1: 1$ & 150 & 1355 & -1 & 10 & - \\
\hline 24 & $-\frac{1}{2}:-\frac{3}{2}: 1$ & 506 & 800 & -3.5 & 20 & - \\
\hline
\end{tabular}

Table 3. Input parameters at GUT scale for the benchmark point chosen for each of the 13 models. We choose the parameters such that in each case we get a maximal contribution from SUSY to muon $(g-2)$.

Also in table 2, model 20 which has the gaugino mass ratio 5/2:-3/2:1 having a higgsino dominated LSP is compatible with all the low energy constraints but only for $A_{0}=-1 \mathrm{TeV}$.

We show the mass spectrum for wino models in table 4, bino models in table 5 and higgsino models in table 6 which satisfy all the low energy constraints listed in the beginning of the section. These are models $1-5,9-11,18-20,22$ and 24 as given in tables 1 and 2. The input parameters for each of the benchmark scenarios are shown in table 3 . The non-universal gaugino models 11 and 19 have been examined in ref. [53]. For models 11, 19 and 24 the parameter space which satisfies all the constraints is restricted in the neighbourhood of the values shown in the benchmark table.

Wino DM. In models $2(-3: 1: 1), 3(-13 / 5: 1: 1)$ and $5(41 / 15: 1: 1)$ the LSP is a wino with mass $1323 \mathrm{GeV}, 1073 \mathrm{GeV}$ and $1189 \mathrm{GeV}$ respectively. In all three models the chargino masses are almost degenerate with the wino LSP masses due to which the chargino co-annihilation processes $\tilde{\chi}_{1}^{0} \tilde{\chi}_{1}^{+} \rightarrow Z W^{+}, c \bar{s}, u \bar{d}$ and $\tilde{\chi}_{1}^{-} \tilde{\chi}_{1}^{+} \rightarrow W^{-} W^{+}$make as much contribution to the relic density in addition as the annihilation channel $\tilde{\chi}_{1}^{0} \tilde{\chi}_{1}^{0} \rightarrow$ $W^{-} W^{+}$. These models come closest to being probed in the direct detection experiments as discussed in section 3.2. In addition models $1(-19 / 5: 1: 1)$ and $4(-22 / 5: 1: 1)$ also show a valid parameter space for $M_{3}=2000-2400 \mathrm{GeV}$, this is because of the well known 
Wino Models

\begin{tabular}{|c|c|c|c|c|c|}
\hline Model no. & 1 & 2 & 3 & 4 & 5 \\
\hline$M_{1}^{G}: M_{2}^{G}: M_{3}^{G}$ & $-\frac{19}{5}: 1: 1$ & $-3: 1: 1$ & $-\frac{13}{5}: 1: 1$ & $-\frac{22}{5}: 1: 1$ & $\frac{41}{15}: 1: 1$ \\
\hline$\tilde{\chi}_{1}^{0}$ & 1673 & 1323 & 1073 & 1688 & 1189 \\
$\tilde{\chi}_{2}^{0}$ & 2160 & 1852 & 1514 & 2120 & 1739 \\
$\tilde{\chi}_{3}^{0}$ & 2167 & 1861 & 1606 & 2129 & 1824 \\
$\tilde{\chi}_{4}^{0}$ & 3490 & 2174 & 1609 & 4071 & 1842 \\
$\tilde{\chi}_{1}^{+}$ & 1673 & 1323 & 1073 & 1688 & 1189 \\
$\tilde{\chi}_{2}^{+}$ & 2168 & 1862 & 1606 & 2129 & 1829 \\
\hline$M_{1}$ & 3538 & 2202 & 1544 & 4129 & 1776 \\
$M_{2}$ & 1632 & 1292 & 1049 & 1647 & 1160 \\
$M_{3}$ & 4144 & 3344 & 2761 & 4175 & 3028 \\
$\mu$ & 2149 & 1847 & 2024 & 2108 & 1818 \\
\hline$\tilde{g}$ & 4262 & 3431 & 2835 & 4305 & 3105 \\
$\tilde{\tau}_{1}$ & 1897 & 1344 & 1076 & 2080 & 1195 \\
$\tilde{\tau}_{2}$ & 2835 & 1782 & 1293 & 3306 & 1482 \\
$\tilde{e}_{R}, \tilde{\mu}_{R}$ & 2846 & 1790 & 1300 & 3318 & 1494 \\
$\tilde{e}_{L}, \tilde{\mu}_{L}$ & 1905 & 1349 & 1081 & 2089 & 1203 \\
$\tilde{t}_{1}$ & 3330 & 2554 & 2056 & 3441 & 2218 \\
$\tilde{t}_{2}$ & 3519 & 2841 & 2364 & 3585 & 2563 \\
$\tilde{b}_{1}$ & 3488 & 2822 & 2344 & 3503 & 2544 \\
$\tilde{b}_{2}$ & 3717 & 2972 & 2467 & 3785 & 2699 \\
$\tilde{u}_{R}$ & 4078 & 3160 & 2586 & 4255 & 2839 \\
$\tilde{u}_{L}$ & 3834 & 3093 & 2574 & 3871 & 2816 \\
\hline$M_{h}($ Higgs $)$ & 124 & 123 & 123 & 124 & 124 \\
$\Omega_{2}^{2}$ & 0.11 & 0.11 & 0.11 & 0.11 & 0.113 \\
$a_{\mu}^{\mathrm{SUSY}}\left(\times 10^{-10}\right)$ & 0.3 & 0.46 & 0.65 & 0.28 & 0.66 \\
\hline
\end{tabular}

Table 4. The SUSY mass spectrum for a chosen benchmark point as suggested in table 3 for each of the wino models which satisfy all the low energy constraints. In addition we also mention the Higgs mass and the relic density in each case. All masses are in GeV.

result that the correct relic density for wino LSP models is achieved at by wino annihilation to W pair by a t-channel chargino exchange with $M_{\mathrm{LSP}} \sim 2 \mathrm{TeV}$ [54].

Of all the wino models only model $8(77 / 5: 1: 1)$ does not have any valid parameter space for the region that we scan. Here, for $M_{3}^{G} \lesssim 1600 \mathrm{GeV}$ the relic density is under abundant while for $M_{3}^{G} \gtrsim 1600$ there is no EWSB.

We have noted that if we allow the larger parameter space for $M_{3}^{G}$, models 1 and 4 allows some parameter space which is consistent with the constraints that we have imposed in our study. It is interesting to mention that for these models to be compatible with the 
correct relic density, $M_{3}^{G}$ needs to be more than $2 \mathrm{TeV}$ in both cases, see figure 2 . We have not extended $M_{3}^{G}$ value beyond $2 \mathrm{TeV}$ for other models as they already qualify to be allowed models for smaller ranges of parameters.

Bino DM. There are three models which have bino LSP as the DM but with very different benchmark spectrum. In model $10(9 / 5: 1: 1)$ the DM is a $934 \mathrm{GeV}$ bino LSP. The chargino mass is close to the LSP mass and chargino coannihilation processes, $\tilde{\chi}_{1}^{0} \tilde{\chi}_{1}^{+} \rightarrow t \bar{b}$; $\tilde{\chi}_{1}^{-} \tilde{\chi}_{1}^{+} \rightarrow t \bar{t}, b \bar{b}$ are important for relic density. In addition the Next to Lightest Supersymmetric Particle (NLSP) mass is close at $970 \mathrm{GeV}$ and the NLSP coannihilation processes, $\tilde{\chi}_{1}^{0} \tilde{\chi}_{2}^{0} \rightarrow b \bar{b}$ and $\tilde{\chi}_{2}^{0} \tilde{\chi}_{2}^{0} \rightarrow b \bar{b}$ makes a significant contribution to the DM annihilation. As a result, the parameters $A_{t}$ and $A_{b}$ significantly affect the parameter space for achieving the correct relic density. This is seen in the top panel of figure 3 with the parameter space for different values of $A_{0}$ being split further apart as compared to figures 1 and 2 .

In model $19(-5: 3: 1)$ the LSP is predominantly bino with higgsino mixture $\left(N_{11}=\right.$ $\left.0.826, N_{13}=0.449, N_{14}=0.338\right)$ of mass $159 \mathrm{GeV}$. The processes $\tilde{\chi}_{1}^{0} \tilde{\chi}_{1}^{0} \rightarrow W^{+} W^{-}, Z Z$ contribute to the relic density.

In model $24(-1 / 2:-3 / 2: 1)$ the LSP is a bino of mass $178 \mathrm{GeV}$ and the main annihilation channel is the stau coannihilation $\tilde{\chi}_{1}^{0} \tilde{\tau} \rightarrow A \tau ; \tilde{\tau} \tilde{\tau} \rightarrow \tau \bar{\tau}, A A ; \tilde{\chi}_{1}^{0} \tilde{\tau} \rightarrow Z \tau$ which are all an order of magnitude larger than the annihilation channel $\tilde{\chi}_{1}^{0} \tilde{\chi}_{1}^{0} \rightarrow \tau \bar{\tau}$. The stau coannihilation channels are boosted up by taking the stau mass $184.5 \mathrm{GeV}$ close to the LSP mass. In addition the models 18 and 22 also show a very small parameter space in the stau coannihilation region. These two models in particular require that the $\tilde{\tau}_{1}$ mass be taken very close to the LSP mass (within $5 \mathrm{GeV}$ ) and in that sense are more fine tuned than the rest of the successful models.

The bino models which do not work in our parameter scan are models 14, 15, 16, 17, $18,21,22,23$ and 25 with their ratios as given in table 2. For these models either the relic density is over abundant or $\tilde{\tau}_{1}$ becomes the LSP or the model is unphysical (tachyonic modes). For model 14, when $m_{0} \leqslant 200 \mathrm{GeV} \tilde{\tau}_{1}$ is the LSP, and when $m_{0}>200 \mathrm{GeV}$ the relic density is over abundant with stau coannihilation dominating in the lower $m_{0}$ range while for $m_{0}>500 \mathrm{GeV}$ the dominant contribution to relic density coming from leptonic channel which is suppressed. In model 15 the correct relic density is achieved through stau coannihilation for $m_{0} \lesssim 200 \mathrm{GeV}$, but the Higgs mass is lighter than the acceptable limit of $122 \mathrm{GeV}$. Whereas for $m_{0} \gtrsim 200 \mathrm{GeV}$ the Higgs mass is in the acceptable range for most of the parameter space but the relic density becomes overabundant with annihilation to leptons dominating the relic density contribution. In addition for $M_{3}^{G}>1 \mathrm{TeV} \tilde{\tau}_{1}$ becomes the LSP. In model 16, for $m_{0}<200 \mathrm{GeV}$ the parameter space is unphysical, and for $m_{0} \geqslant 200$ the relic density is over abundant with the dominant annihilation channels into $\tau \bar{\tau}$ and $b \bar{b}$. Model 17 is similar to model 15 , however in case of model 17 the $\tilde{\tau}_{1}$ mass is below LEP limit for $m_{0}<200 \mathrm{GeV}$. Model 21 is similar to model 18, but is ruled out because of the higgs mass constraint. For model 22 , at low $m_{0}$ values below $400 \mathrm{GeV}$ the LSP is $\tilde{\tau}_{1}$. At higher values of $m_{0}$ the bino LSP which gives overabundant relic density crosses over to higgsino dominated LSP as $M_{3}$ increases. For the region with higgsino dominated LSP the relic density is again overabundant with the main contribution to relic density coming from 

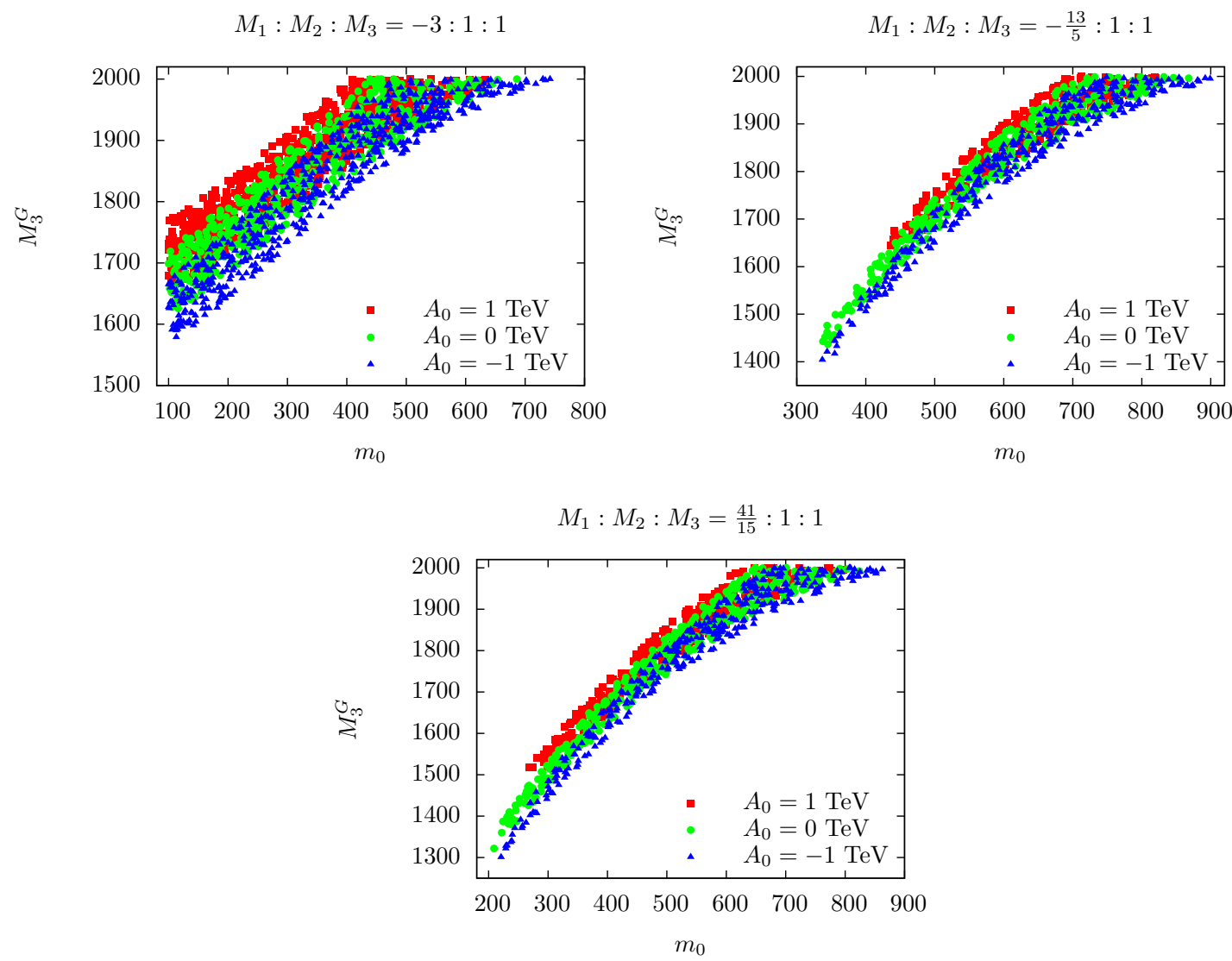

Figure 1. The allowed parameter space satisfying all the low energy constraints as listed in the text except muon $(g-2)$ for heavy wino DM models with the GUT scale the gaugino mass ratios as mentioned on top of each panel. The choice of other parameters are $\tan \beta=10, \operatorname{sgn}(\mu) \equiv$ $+v e$ (positive). For model $2(-3: 1: 1)$ the allowed mass range for $m_{0}$ is $\sim 100-700 \mathrm{GeV}$ for $A_{0}=0,1 \mathrm{TeV}$ with $M_{3}^{G}$ ranging from $\sim 1600-2000 \mathrm{GeV}$, whereas for $A_{0}=-1 \mathrm{TeV}, m_{0}$ ranges between $\sim 100-750 \mathrm{GeV}$ with $M_{3}^{G}$ between $\sim 1550-2000 \mathrm{GeV}$. For model $3(-13 / 5: 1: 1)$ the allowed mass range for $m_{0}$ is $\sim 300-900 \mathrm{GeV}$ with $M_{3}^{G}$ between $\sim 1400-2000 \mathrm{GeV}$ for $A_{0}=0,-1 \mathrm{TeV}$, but for $A_{0}=1 \mathrm{TeV}, m_{0}$ ranges between $\sim 400-800 \mathrm{GeV}$ with $M_{3}^{G}$ between $\sim 1600-2000 \mathrm{GeV}$. For model $5(41 / 15: 1: 1)$ the allowed mass range for $m_{0}$ is $\sim 200-900 \mathrm{GeV}$ with $M_{3}^{G}$ between $\sim 1300-2000 \mathrm{GeV}$ for $A_{0}=0,-1 \mathrm{TeV}$, but for $A_{0}=1 \mathrm{TeV}, m_{0}$ ranges between $\sim 300-800 \mathrm{GeV}$ with $M_{3}^{G}$ between $\sim 1450-2000 \mathrm{GeV}$.

coannihilation channel. Model 25 behaves similar to model 22 , however for low $m_{0}$ values below $300 \mathrm{GeV}$ the correct relic density is achieved through stau coannihilation however the higgs mass constraint is not satisfied. While for higher values of $m_{0}$ beyond $300-400 \mathrm{GeV}$, the higgs mass constraint does get satisfied but the relic density remains overabundant even in stau-coannihilation region of the parameter space.

Higgsino DM. In model $9(10: 2: 1)$ the LSP is a higgsino and the relic density is via the chargino coannihilation processes $\tilde{\chi}_{1}^{0} \tilde{\chi}_{1}^{+} \rightarrow u \bar{d}, c \bar{s}$. The NLSP mass is close to the LSP mass and the NLSP coannihilation $\tilde{\chi}_{2}^{0} \tilde{\chi}_{1}^{+} \rightarrow u \bar{d}, c \bar{s}$ also contributes to the relic density. 

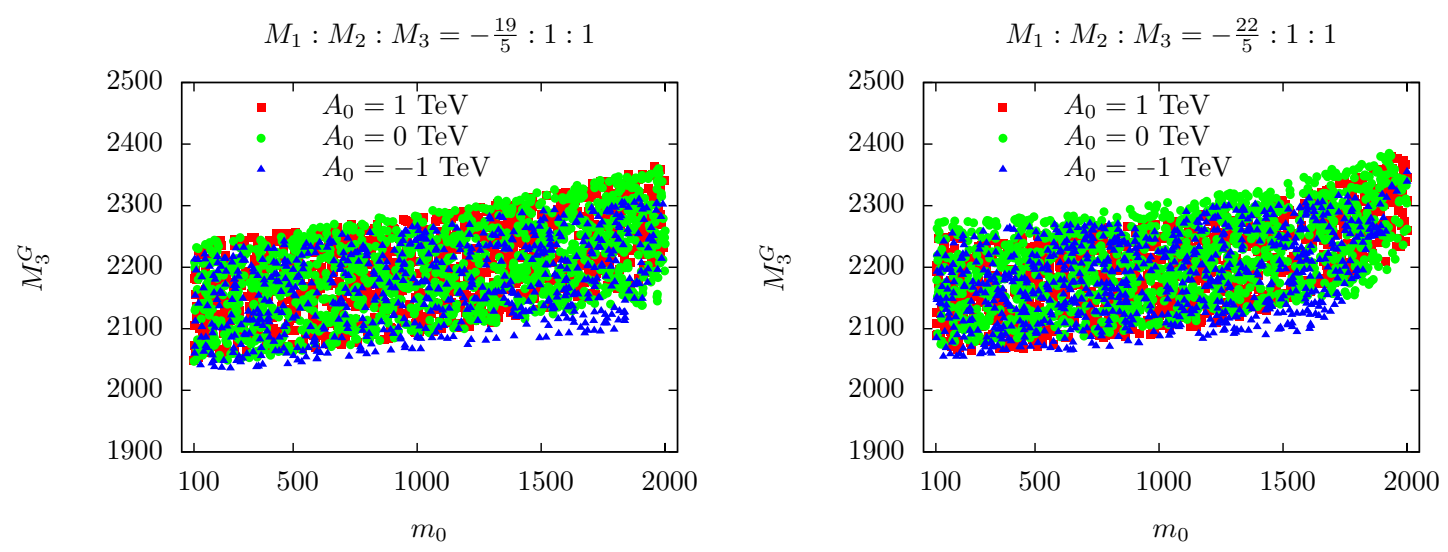

Figure 2. The allowed parameter space for heavy wino models $1(-19 / 5: 1: 1)$ and $4(-22 / 5: 1: 1)$ shown in the left and right panels respectively. We extend the scan range for $M_{3}^{G}$ upto $3 \mathrm{TeV}$ for these two models. The allowed mass range for $M_{3}^{G}$ lies between $\sim 2.0-2.4 \mathrm{TeV}$ while for $m_{0}$ it covers the entire range of our scan from $100-2000 \mathrm{GeV}$.

In model $11(-1 / 5: 3: 1)$ the LSP is a higgsino with mass $1015 \mathrm{GeV}$ and the relic density is via the same chargino coannihilation processes as in model 9 including the NLSP coannihilation contribution.

In model $20(5 / 2:-3 / 2: 1)$ the LSP is a higgsino of mass $1507 \mathrm{GeV}$ and the contributions to the relic density are due to the chargino coannihilation $\tilde{\chi}_{1}^{0} \tilde{\chi}_{1}^{+} \rightarrow t \bar{b} ; \tilde{\chi}_{1}^{-} \tilde{\chi}_{1}^{+} \rightarrow t \bar{t}, b \bar{b}$ in addition to the main annihilation channel $\tilde{\chi}_{1}^{0} \tilde{\chi}_{1}^{0} \rightarrow b \bar{b}, t \bar{t}$. The NLSP mass is close to the LSP mass and the NLSP coannihilation $\tilde{\chi}_{2}^{0} \tilde{\chi}_{1}^{+} \rightarrow t \bar{b}$ also contributes to the relic density. This model gives the correct relic density for $A_{0} \sim-1 \mathrm{TeV}$.

The failed higgsino models are models $6(122 / 5: 1: 1), 7(-101 / 10:-3 / 2: 1), 12(1$ : $35 / 9: 1)$ and $13(1:-5: 1)$. All of these models fail because the spectrum is unphysical or the higgs sector is unstable. In model 6 for $m_{0} \leq 1200 \mathrm{GeV}$ the spectrum contains tachyonic modes, while for $m_{0} \geq 1200 \mathrm{GeV}$ there is no EWSB and as $M_{3}$ increases one again encounters tachyonic modes in the spectrum. In model 7 the relic density is under abundant for $M_{3}^{G}<1.3 \mathrm{TeV}$ while for higher values of $M_{3}^{G}$ there is no EWSB. Model 12 behaves very similar to model 6 and so fails for the same reasons. For model 13, there is no EWSB below a certain value of $M_{3}$ for a given $m_{0}$, and this value increases with $m_{0}$. Above this value of $M_{3}$ some of the scalar modes are tachyonic.

\subsection{Direct detection constraints}

The elastic scattering of neutralinos with nucleons which results in spin-independent cross section is by Higgs exchange. The Higgs coupling to the lightest neutralino depends upon the product of the higgsino and the gaugino fraction of the neutralino. Pure bino DM therefore easily evade the direct detection limits from XENON100 [42]. In model 24 ( $5 / 2:-3 / 2: 1$ ) with a $176 \mathrm{GeV}$ bino DM evades the XENON100 bound but may be probed in Xenon 1000 as shown in figure 7 . While model $10(9 / 5: 1: 1)$ which gives a $\sim 1 \mathrm{TeV}$ bino DM also easily evades the XENON100 bound as shown in figure 6 . In model 

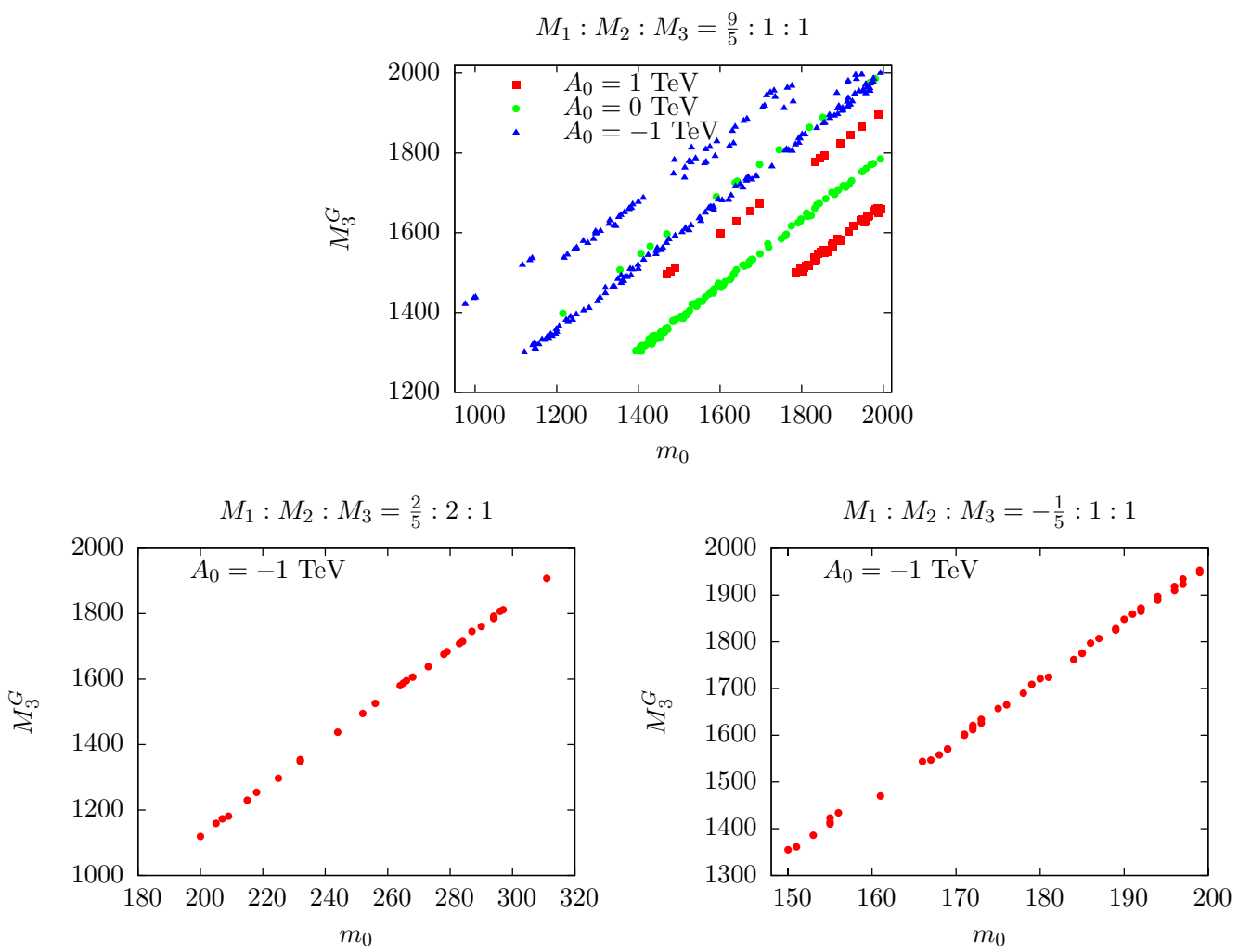

Figure 3. Same as figure 1 but for heavy bino DM models $10(9 / 5: 1: 1), 18(2 / 5: 2: 2)$ and $22(-1 / 5: 1: 1)$. As before all low energy constraints except muon $(g-2)$ are satisfied. For model $10(9 / 5: 1: 1)$ shown in the top panel, the allowed mass range for $m_{0}$ is $\sim 1200-2000 \mathrm{GeV}$ with $M_{3}^{G}$ between $\sim 1300-1900 \mathrm{GeV}$ for $A_{0}=0 \mathrm{TeV}$, while for $A_{0}=1 \mathrm{TeV}, m_{0}$ ranges between $\sim 1500-2000 \mathrm{GeV}$ with $M_{3}^{G}$ between $\sim 1500-1900 \mathrm{GeV}$ and finally for $A_{0}=-1 \mathrm{TeV} m_{0}$ lies between $\sim 1000-2000 \mathrm{GeV}$ and $M_{3}^{G}$ between $\sim 1300-2000 \mathrm{GeV}$. The models $18(2 / 5: 2: 2)$ and $22(-1 / 5: 1: 1)$ shown in the bottom left and right panels respectively, have a small parameter space and are more fine-tuned than the other models studied here. We show the result for $A_{0}=-1 \mathrm{TeV}$. For model $18(2 / 5: 2: 2)$ the allowed mass range for $m_{0}$ is $\sim 200-320 \mathrm{GeV}$ and $M_{3}^{G}$ ranges between $\sim 1100-1900 \mathrm{GeV}$. For model $22(-1 / 5: 1: 1)$ the allowed mass range for $m_{0}$ is $\sim 150-200 \mathrm{GeV}$ while for $M_{3}^{G}$ it is $\sim 1300-1950 \mathrm{GeV}$.

$19(-5: 3: 1)$ where the $159 \mathrm{GeV}$ LSP is predominantly bino with a higgsino mixture $\left(N_{11}=0.826, N_{13}=0.449, N_{14}=0.338\right)$ has a SI cross section $\sim 1.01 \times 10^{-8} \mathrm{pb}$ and is incompatible with the XENON100 exclusion limits.

The Spin Independent (SI) cross section for model $20(5 / 2:-3 / 2: 1)$ which is a $1.5 \mathrm{TeV}$ higgsino DM also evades the XENON100 bound easily as shown in figure 6 as the gaugino fraction is small. Similarly model $11(-1 / 5: 3: 1)$ with a $1 \mathrm{TeV}$ wino DM has a SI cross section $\sim 7 \times 10^{-11} \mathrm{pb}$ and evades the XENON100 bound.

The three wino dark matter models $2(-3: 1: 1), 3(-13 / 5: 1: 1)$ and $5(41 / 15: 1: 1)$ with a small mixing of higgsino have larger SI cross sections as shown in figure 5 . These wino DM models may be within the reach of XENON1T [40] and Super-CDMS [41] experiments. 
Bino Models

\begin{tabular}{|c|c|c|c|c|c|}
\hline Model no. & 10 & 18 & 19 & 22 & 24 \\
\hline$M_{1}^{G}: M_{2}^{G}: M_{3}^{G}$ & $\frac{9}{5}: 1: 1$ & $\frac{2}{5}: 2: 1$ & $-5: 3: 1$ & $-\frac{1}{5}: 1: 1$ & $-\frac{1}{2}:-\frac{3}{2}: 1$ \\
\hline$\tilde{\chi}_{1}^{0}$ & 934.3 & 188.6 & 159.2 & 131.2 & 177.6 \\
\hline$\tilde{\chi}_{2}^{0}$ & 970.4 & 1252 & 202.6 & 1103 & 976.4 \\
\hline$\tilde{\chi}_{3}^{0}$ & 1551 & 1259 & 219.6 & 1696 & 1523 \\
\hline$\tilde{\chi}_{4}^{0}$ & 1558 & 1828 & 4219 & 1699 & 1528 \\
\hline$\tilde{\chi}_{1}^{+}$ & 970.1 & 1252 & 1999 & 1103 & 976.4 \\
\hline$\tilde{\chi}_{2}^{+}$ & 1557 & 1828 & 4219 & 1699 & 1528 \\
\hline$M_{1}$ & 943.6 & 190.1 & 174.4 & 133.2 & 177.9 \\
\hline$M_{2}$ & 943.1 & 1803 & 4194 & 1076 & 981.4 \\
\hline$M_{3}$ & 2497 & 2344 & 3494 & 2824 & 1771 \\
\hline$\mu$ & 1545 & 1251 & 1943 & 1691 & 1521 \\
\hline$\tilde{g}$ & 2596 & 2420 & 3644 & 2883 & 1805 \\
\hline$\tilde{\tau}_{1}$ & 1253 & 195.3 & 740.6 & 139.1 & 184.5 \\
\hline$\tilde{\tau}_{2}$ & 1301 & 1419 & 3310 & 857.6 & 861.2 \\
\hline$\tilde{e}_{R}, \tilde{\mu}_{R}$ & 1271 & 259.4 & 797.3 & 184.6 & 528.0 \\
\hline$\tilde{e}_{L}, \tilde{\mu}_{L}$ & 1303 & 1424 & 3316 & 861 & 926.6 \\
\hline$\tilde{t}_{1}$ & 1847 & 1391 & 1559 & 1959 & 775.7 \\
\hline$\tilde{t}_{2}$ & 2264 & 2258 & 4039 & 2398 & 1440 \\
\hline$\tilde{b}_{1}$ & 2250 & 2049 & 3089 & 2386 & 1404 \\
\hline$\tilde{b}_{2}$ & 2412 & 2249 & 4033 & 2465 & 1473 \\
\hline$\tilde{u}_{R}$ & 2467 & 2069 & 3125 & 2480 & 1632 \\
\hline$\tilde{u}_{L}$ & 2515 & 2482 & 4439 & 2606 & 1800 \\
\hline$M_{h}$ (Higgs) & 123 & 123 & 125 & 122 & 124 \\
\hline$\Omega h^{2}$ & 0.12 & 0.11 & 0.12 & 0.11 & 0.12 \\
\hline$a_{\mu}^{\text {SUSY }}\left(\times 10^{-10}\right)$ & 0.79 & 0.16 & 0.28 & 1.0 & 2.65 \\
\hline
\end{tabular}

Table 5. The SUSY mass spectrum for a chosen benchmark point as suggested in table 3 for each of the bino models which satisfy all the low energy constraints. In addition we also mention the Higgs mass and the relic density in each case. All masses are in GeV.

\section{Muon $(g-2)$}

It has long been recognised that to explain the discrepancy between experiment and SM prediction for muon anomalous magnetic moment from a SUSY contribution would require a light mass spectrum on the gauginos and the sleptons $[55,56]$ which would put a severe restriction on the SUSY models.

The SUSY contribution to muon $(g-2)$ for light binos is through the bino-smuon loop $[57,58]$ so the largest $a_{\mu}^{\mathrm{SUSY}}=2.65 \times 10^{-10}[37-39,50]$ comes from model 24 which has the lightest LSP $\left(177 \mathrm{GeV}\right.$ bino) and slepton spectrum. In model $24\left(M_{1}^{G}: M_{2}^{G}\right.$ : 

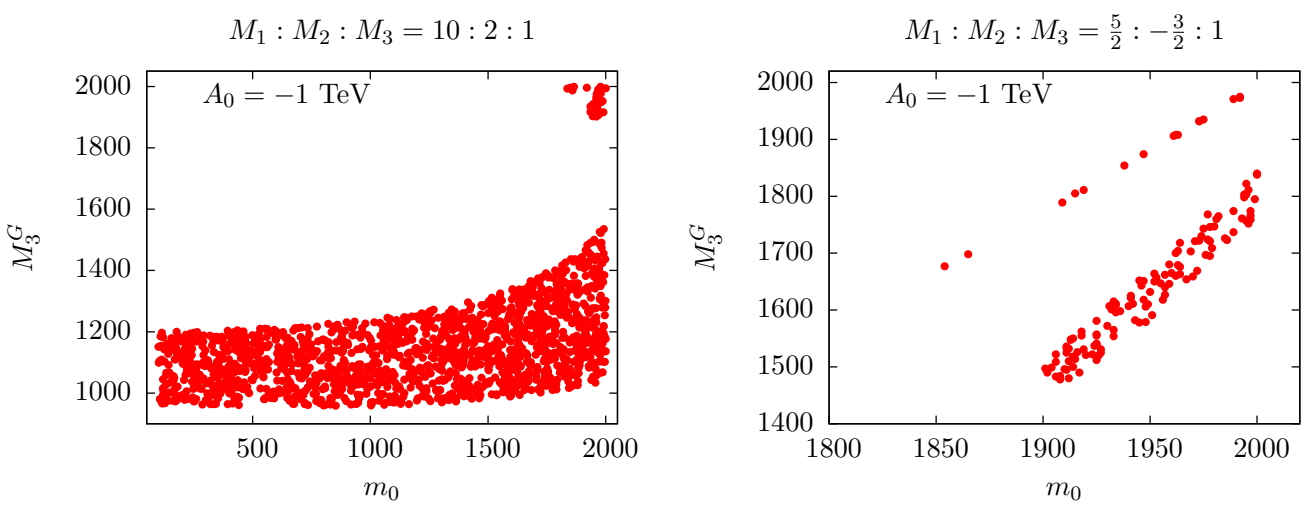

Figure 4. The allowed parameter space satisfying the low energy constraints except muon $(g-2)$ for heavy higgsino DM models $9(10: 2: 1)$ and model $20(5 / 2:-3 / 2: 1)$. All parameters are chosen as in figure 1 except $A_{0}=-1 \mathrm{TeV}$. For model $9(10: 2: 1)$ the allowed mass range for $m_{0}$ spans the entire range of scan from $100-2000 \mathrm{GeV}$ with $M_{3}^{G}$ between $\sim 950-1550 \mathrm{GeV}$. For model $20(5 / 2:-3 / 2: 1)$ the allowed mass range for $m_{0}$ is $\sim 1850-2000 \mathrm{GeV}$ with $M_{3}^{G}$ between $\sim 1400-2000 \mathrm{GeV}$. These models do not work for $A_{0}=0,1 \mathrm{TeV}$.

$M_{3}^{G}=-1 / 2:-3 / 2: 1$ ) it would have been easy to adjust the smuon mass (through $m_{0}^{G}$ ) and the bino mass through $M_{3}^{G}$ (as $M_{1}^{G}$ is related to $M_{3}^{G}$ ) to get a much larger contribution to muon $(g-2)$. However the relic density of bino DM in model 24 depends on the stau coannihilation which has to be close to the bino DM mass of $177 \mathrm{GeV}$ which is again determined by the universal scalar mass $m_{0}$. So demanding the correct relic density results in a less than optimum contribution to the muon $(g-2)$ in this model. In table 2 one can note that the gaugino mass ratio referred to here as model 24 , can arise from three possible breaking patterns of $\mathrm{SO}(10)$, each of them through a different intermediate symmetry group. It will be interesting to see if we distinguish intermediate scale separately than the unification scale then muon $(g-2)$ is further improved or not. We have kept this issue for our further publication. The gaugino mass ratio of model 24 has been studied in ref. [59] in the context of Yukawa unification in $\mathrm{SO}(10)$, but in the benchmark models examined in [59] the SUSY contribution to muon $g-2$ is an order of magnitude smaller than the benchmark parameters for model 24 shown in table 5 .

In this paper we have chosen a single non-singlet scalar for giving masses to the gauginos. By choosing a the gaugino masses to arise from more than one scalar representation like $1+24,1+75$ and $1+200$ of $\mathrm{SU}(5)[14,16,60]$ it is possible to explain muon $(g-2)$ from SUSY contributions along with the Planck-WMAP relic density [61]. It has been noted [62] that in a mSUGRA model the gaugino mass ratio $M_{1}: M_{2}: M_{3}=1: 1: 10$ at the GUT scale gives the required muon $(g-2)$, but in this paper we see that this gaugino ratio does not arise from any of the GUT breaking patterns if one considers one non-singlet Higgs representation for generating the gaugino masses.

If one were to have non-universal scalar masses [27, 28] it may be possible to adjust the stau mass to control the relic relic density and the smuon mass to fit muon $(g-2)$ using a single scalar representation for getting non-universal gaugino masses. 
Higgsino Models

\begin{tabular}{|c|c|c|c|}
\hline Model no. & 9 & 11 & 20 \\
\hline$M_{1}^{G}: M_{2}^{G}: M_{3}^{G}$ & $10: 2: 1$ & $-\frac{1}{5}: 3: 1$ & $\frac{5}{2}:-\frac{3}{2}: 1$ \\
\hline$\tilde{\chi}_{1}^{0}$ & 1006 & 1015 & 1507 \\
\hline$\tilde{\chi}_{2}^{0}$ & 1013 & 1016 & 1510 \\
\hline$\tilde{\chi}_{3}^{0}$ & 1584 & 3791 & 1958 \\
\hline$\tilde{\chi}_{4}^{0}$ & 4258 & 4093 & 2230 \\
\hline$\tilde{\chi}_{1}^{+}$ & 1007 & 1015 & 1507 \\
\hline$\tilde{\chi}_{2}^{+}$ & 1584 & 4093 & 2230 \\
\hline$M_{1}$ & 4294 & 3797 & 1969 \\
\hline$M_{2}$ & 1549 & 4051 & 2175 \\
\hline$M_{3}$ & 2023 & 3361 & 3570 \\
\hline$\mu$ & 1002 & 1000 & 1495 \\
\hline$\tilde{g}$ & 2164 & 3585 & 3772 \\
\hline$\tilde{\tau}_{1}$ & 2138 & 3181 & 2455 \\
\hline$\tilde{\tau}_{2}$ & 3537 & 3779 & 2619 \\
\hline$\tilde{e}_{R}, \tilde{\mu}_{R}$ & 3554 & 3620 & 2473 \\
\hline$\tilde{e}_{L}, \tilde{\mu}_{L}$ & 2152 & 3181 & 2628 \\
\hline$\tilde{t}_{1}$ & 1767 & 2309 & 2910 \\
\hline$\tilde{t}_{2}$ & 2254 & 3716 & 3625 \\
\hline$\tilde{b}_{1}$ & 1782 & 2812 & 3617 \\
\hline$\tilde{b}_{2}$ & 2125 & 3726 & 3656 \\
\hline$\tilde{u}_{R}$ & 2945 & 4051 & 3785 \\
\hline$\tilde{u}_{L}$ & 2226 & 4662 & 3988 \\
\hline$M_{h}$ (Higgs) & 124 & 127 & 122 \\
\hline$\Omega h^{2}$ & 0.11 & 0.12 & 0.11 \\
\hline$a_{\mu}^{\mathrm{SUSY}}\left(\times 10^{-10}\right)$ & 0.44 & 0.47 & 0.24 \\
\hline
\end{tabular}

Table 6. The SUSY mass spectrum for a chosen benchmark point as suggested in table 3 for each of the higgsino models which satisfy all the low energy constraints. In addition we also mention the Higgs mass and the relic density in each case. All masses are in GeV.

\section{Conclusions}

In this paper we have exhaustively analysed all possible non-universal gaugino mass models that arise from $\mathrm{SU}(5), \mathrm{SO}(10), \mathrm{E}(6)$ SUSY GUT models. The underlying assumption is that the full gauge symmetry is broken to the SM symmetry group at the GUT scale itself, i.e., the intermediate scales are same as the GUT scale. We have considered all these models in its minimal versions, i.e., we have not probed the effect of the presence of multiple non-singlet scalars. If one considers that the contribution to the effective gaugino mass ratios are outcome of the contributions from more than one scalar field with the 

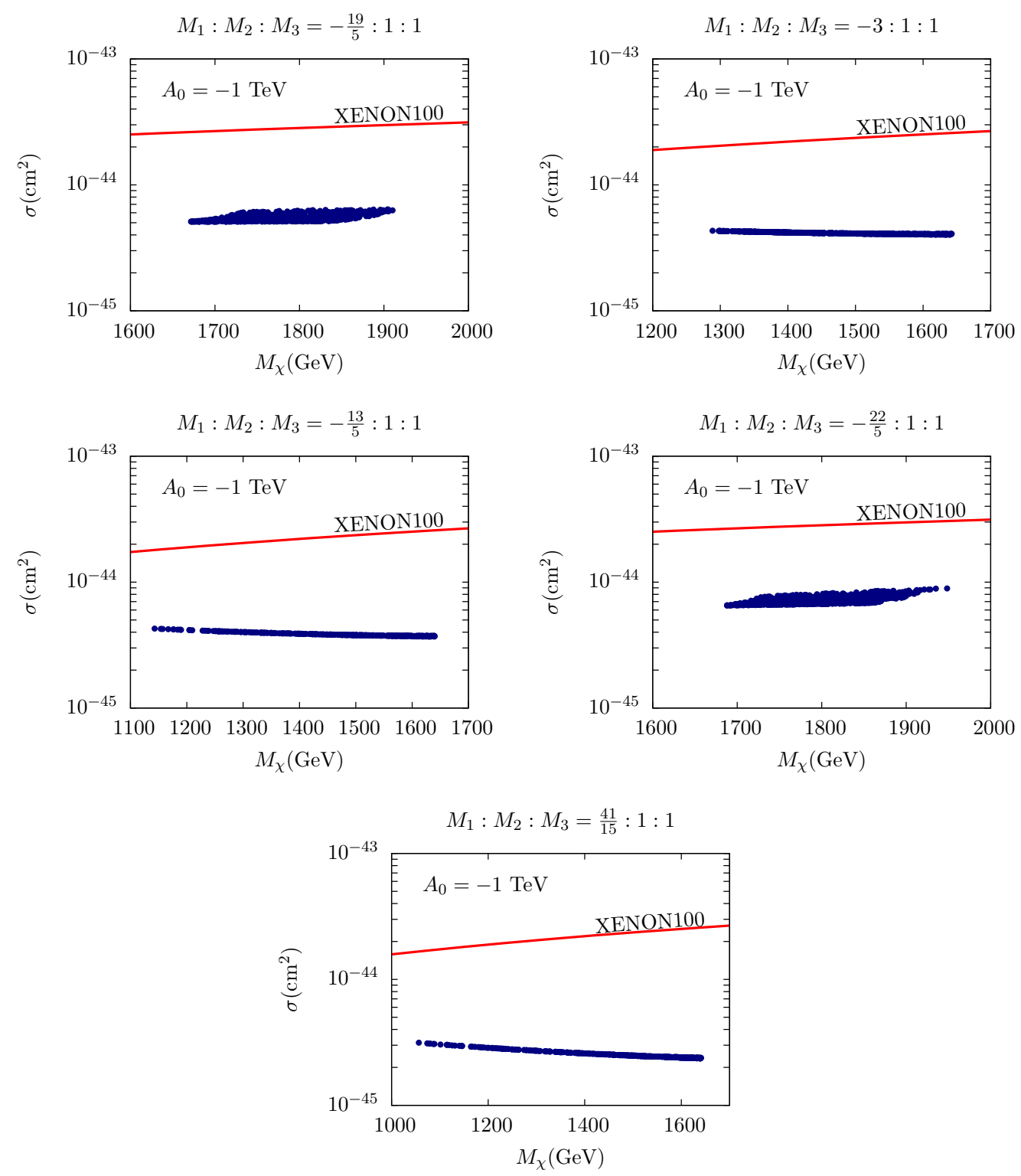

Figure 5. The direct detection spin independent proton-DM scattering cross section plotted with the constraint from XENON100 [42]. These plots show selected points for the heavy wino models satisfying all the low energy constraints considered here, except for muon $(g-2)$. These heavy wino models satisfy the XENON100 constraint.

introduction of one or more free parameters, the the unique group theoretic characteristics of the models are lost. Thus we restrict ourselves to the minimal versions (from the point of number of free parameters) of the non-universal gaugino models. We have shown different models predict different kind of LSP compositions. Thus the contributions to the relic density from such models are discriminated. We have performed a comparative study among such models using the collider constraints, lightest Higgs mass and the relic density. We also emphasise the importance of muon $(g-2)$ and briefly argue why model 

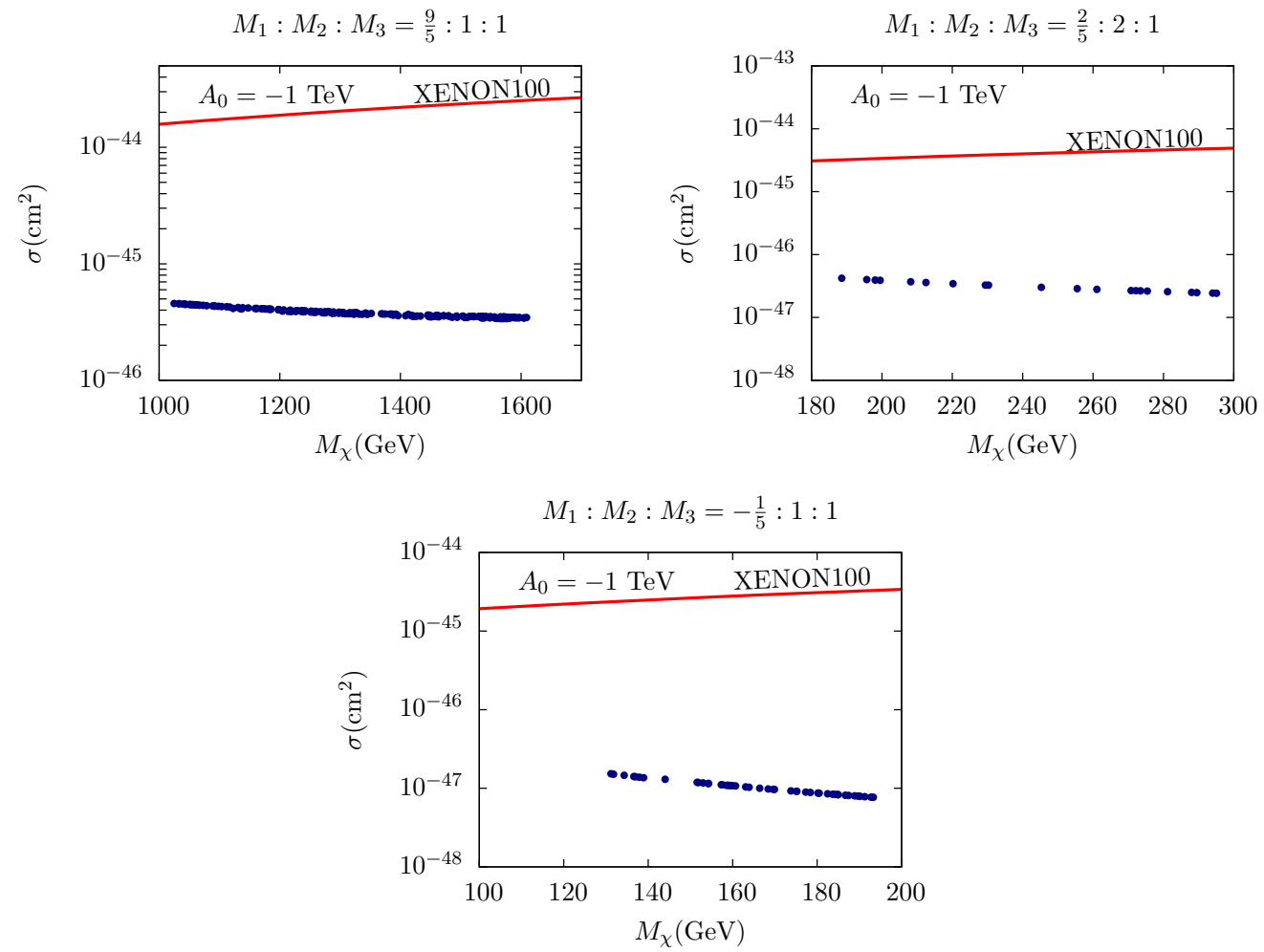

Figure 6. The direct detection spin independent proton-DM scattering cross section plotted with the constraint from XENON100 [42]. These plots show selected points for bino models satisfying all the low energy constraints considered here, except for muon $(g-2)$. These bino models satisfy the XENON100 constraint.
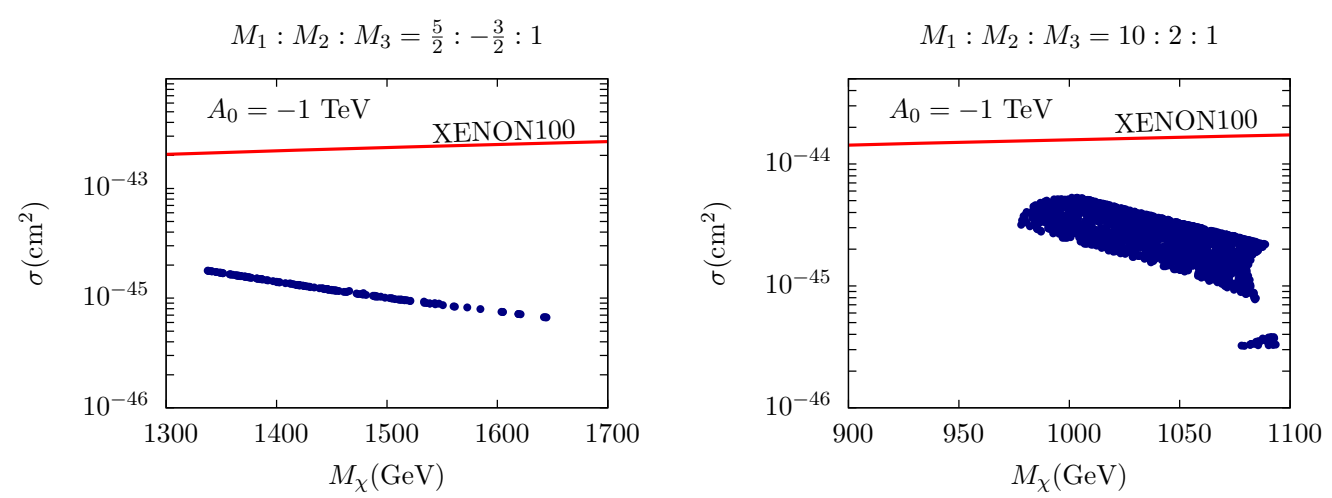

Figure 7. The direct detection spin independent proton-DM scattering cross section plotted with the constraint from XENON100 [42]. These plots show selected points for the heavy higgsino models satisfying all the low energy constraints considered here, except for muon $(g-2)$. These heavy higgsino models satisfies the XENON100 constraint.

$24\left(M_{1}^{G}: M_{2}^{G}: M_{3}^{G}=-1 / 2:-3 / 2: 1\right)$ is the best candidate among other models in the context of muon $(g-2)$ contribution. We also check the status of bino-, wino-, and higgisno- dominated models in the context of Direct detection constraints. The model 19 $(-5: 3: 1)$ is ruled out by XENON100 [42]. The three models $2(-3: 1: 1), 3(-13 / 5: 1: 1)$ 
and $5(41 / 5: 1: 1)$ where the dark matter is a TeV scale wino can be probed in upcoming direct detection experiments like XENON1T [40] and Super-CDMS [41].

Finally we would like to comment on the impact of the insertions of the intermediate scales. In supersymmetric grand unified theories in case of one step breaking the usual trend of the intermediate scale is to lie around the unification scale, see [46]. Thus we expect that the ratios at the GUT scale will not change visibly by the new set of RGEs from intermediate scale to the unification scale. But in case of two step symmetry breaking the second intermediate scale can as low as $100 \mathrm{TeV}$ [46] within a proper unification frame work. If the second intermediate scale is low enough then a new set of RGEs will change the gaugino mass ratios at the GUT scale widely. We are looking into this issue in detail and postpone and will present the results in a future publication.

\section{Acknowledgments}

Work of JC is supported by Department of Science \& Technology, Government of INDIA under the Grant Agreement number IFA12-PH-34 (INSPIRE Faculty Award).

Open Access. This article is distributed under the terms of the Creative Commons Attribution License (CC-BY 4.0), which permits any use, distribution and reproduction in any medium, provided the original author(s) and source are credited.

\section{References}

[1] J.R. Ellis, K. Enqvist, D.V. Nanopoulos and K. Tamvakis, Gaugino Masses and Grand Unification, Phys. Lett. B 155 (1985) 381 [INSPIRE].

[2] M. Drees, Phenomenological Consequences of $N=1$ Supergravity Theories With Nonminimal Kinetic Energy Terms for Vector Superfields, Phys. Lett. B 158 (1985) 409 [InSPIRE].

[3] G. Anderson, C. H. Chen, J. F. Gunion, J. D. Lykken, T. Moroi and Y. Yamada, Motivations for and implications of nonuniversal GUT scale boundary conditions for soft SUSY breaking parameters, eConf C 960625 (1996) SUP107 [hep-ph/9609457] [INSPIRE].

[4] G. Anderson, H. Baer, C.-h. Chen and X. Tata, The Reach of Fermilab Tevatron upgrades for SU(5) supergravity models with nonuniversal gaugino masses, Phys. Rev. D 61 (2000) 095005 [hep-ph/9903370] [INSPIRE].

[5] K. Huitu, Y. Kawamura, T. Kobayashi and K. Puolamaki, Phenomenological constraints on SUSY SU(5) GUTs with nonuniversal gaugino masses, Phys. Rev. D 61 (2000) 035001 [hep-ph/9903528] [INSPIRE].

[6] A. Corsetti and P. Nath, Gaugino mass nonuniversality and dark matter in SUGRA, strings and D-brane models, Phys. Rev. D 64 (2001) 125010 [hep-ph/0003186] [INSPIRE].

[7] U. Chattopadhyay and D. Roy, Higgsino dark matter in a SUGRA model with nonuniversal gaugino masses, Phys. Rev. D 68 (2003) 033010 [hep-ph/0304108] [INSPIRE].

[8] J. Chakrabortty and A. Raychaudhuri, A note on dimension-5 operators in GUTs and their impact, Phys. Lett. B 673 (2009) 57 [arXiv:0812.2783] [INSPIRE].

[9] S.P. Martin, Non-universal gaugino masses from non-singlet F-terms in non-minimal unified models, Phys. Rev. D 79 (2009) 095019 [arXiv:0903.3568] [INSPIRE]. 
[10] S. Bhattacharya and J. Chakrabortty, Gaugino mass non-universality in an $\mathrm{SO}(10)$ supersymmetric Grand Unified Theory: low-energy spectra and collider signals, Phys. Rev. D 81 (2010) 015007 [arXiv:0903.4196] [INSPIRE].

[11] J. Chakrabortty and A. Raychaudhuri, Dimension-5 operators and the unification condition in $\mathrm{SO}(10)$ and $\mathrm{E}_{6}$, arXiv: 1006.1252 [INSPIRE].

[12] D. Cerdeno and C. Muñoz, Neutralino dark matter in supergravity theories with non-universal scalar and gaugino masses, JHEP 10 (2004) 015 [hep-ph/0405057] [INSPIRE].

[13] K. Huitu, J. Laamanen, P.N. Pandita and S. Roy, Phenomenology of non-universal gaugino masses in supersymmetric grand unified theories, Phys. Rev. D 72 (2005) 055013 [hep-ph/0502100] [INSPIRE].

[14] S. King, J. Roberts and D. Roy, Natural dark matter in SUSY GUTs with non-universal gaugino masses, JHEP 10 (2007) 106 [arXiv:0705.4219] [INSPIRE].

[15] K. Huitu et al., Search for Higgs Bosons in SUSY Cascades in CMS and Dark Matter with Non-universal Gaugino Masses, Eur. Phys. J. C 58 (2008) 591 [arXiv:0808.3094] [INSPIRE].

[16] U. Chattopadhyay, D. Das and D. Roy, Mixed Neutralino Dark Matter in Nonuniversal Gaugino Mass Models, Phys. Rev. D 79 (2009) 095013 [arXiv:0902.4568] [InSPIRE].

[17] D. Feldman, Z. Liu and P. Nath, Gluino NLSP, Dark Matter via Gluino Coannihilation and LHC Signatures, Phys. Rev. D 80 (2009) 015007 [arXiv:0905.1148] [INSPIRE].

[18] I. Gogoladze, R. Khalid and Q. Shafi, Coannihilation Scenarios and Particle Spectroscopy in $\mathrm{SU}(4)(c) \times \mathrm{SU}(2)(L) \times \mathrm{SU}(2)(R)$, Phys. Rev. D $80(2009) 095016$ [arXiv:0908.0731] [INSPIRE].

[19] M. Holmes and B.D. Nelson, Non-Universal Gaugino Masses, CDMS and the LHC, Phys. Rev. D 81 (2010) 055002 [arXiv: 0912.4507] [InSPIRE].

[20] N. Okada, S. Raza and Q. Shafi, Particle Spectroscopy of Supersymmetric SO(10) with Non-Universal Gaugino Masses, Phys. Rev. D 84 (2011) 095018 [arXiv:1107.0941] [INSPIRE].

[21] N. Bhattacharyya, A. Choudhury and A. Datta, Low mass neutralino dark matter in mSUGRA and more general models in the light of LHC data, Phys. Rev. D 84 (2011) 095006 [arXiv: 1107.1997] [INSPIRE].

[22] S. Amsel, K. Freese and P. Sandick, Probing EWSB Naturalness in Unified SUSY Models with Dark Matter, JHEP 11 (2011) 110 [arXiv:1108.0448] [INSPIRE].

[23] I. Gogoladze, S. Raza and Q. Shafi, Neutralino-Sbottom Coannihilation in SU(5), JHEP 03 (2012) 054 [arXiv:1111.6299] [INSPIRE].

[24] J.E. Younkin and S.P. Martin, Non-universal gaugino masses, the supersymmetric little hierarchy problem and dark matter, Phys. Rev. D 85 (2012) 055028 [arXiv:1201.2989] [INSPIRE].

[25] H. Baer, I. Gogoladze, A. Mustafayev, S. Raza and Q. Shafi, Sparticle mass spectra from SU(5) SUSY GUT models with b- $\tau$ Yukawa coupling unification, JHEP 03 (2012) 047 [arXiv: 1201.4412] [INSPIRE].

[26] S. Caron, J. Laamanen, I. Niessen and A. Strubig, Higgs and non-universal gaugino masses: no SUSY signal expected yet?, JHEP 06 (2012) 008 [arXiv:1202.5288] [INSPIRE].

[27] D. Miller and A. Morais, Supersymmetric SU(5) Grand Unification for a Post Higgs Boson Era, JHEP 10 (2013) 226 [arXiv:1307.1373] [INSPIRE]. 
[28] M. Badziak, M. Olechowski and S. Pokorski, Light staus and enhanced Higgs diphoton rate with non-universal gaugino masses and $\mathrm{SO}(10)$ Yukawa unification, JHEP 10 (2013) 088 [arXiv: 1307.7999] [INSPIRE].

[29] T. Han, Z. Liu and A. Natarajan, Dark matter and Higgs bosons in the MSSM, JHEP 11 (2013) 008 [arXiv: 1303.3040] [INSPIRE].

[30] S. Bhattacharya, S. Chakdar, K. Ghosh and S. Nandi, Non-universal SUGRA at LHC: Prospects and Discovery Potential, Phys. Rev. D 89 (2014) 015004 [arXiv:1309.0036] [INSPIRE].

[31] ATLAS collaboration, Observation of a new particle in the search for the Standard Model Higgs boson with the ATLAS detector at the LHC, Phys. Lett. B 716 (2012) 1 [arXiv: 1207.7214] [INSPIRE].

[32] CMS collaboration, Observation of a new boson at a mass of $125 \mathrm{GeV}$ with the CMS experiment at the LHC, Phys. Lett. B 716 (2012) 30 [arXiv:1207.7235] [INSPIRE].

[33] WMAP collaboration, G. Hinshaw et al., Nine-Year Wilkinson Microwave Anisotropy Probe (WMAP) Observations: Cosmological Parameter Results, Astrophys. J. Suppl. 208 (2013) 19 [arXiv: 1212.5226] [INSPIRE].

[34] Planck collaboration, P. Ade et al., Planck 2013 results. XVI. Cosmological parameters, arXiv: 1303.5076 [INSPIRE].

[35] Heavy Flavor Averaging Group collaboration, Y. Amhis et al., Averages of B-Hadron, C-Hadron and tau-lepton properties as of early 2012, arXiv:1207.1158 [INSPIRE].

[36] CMS collaboration, Search for B(s) and B to dimuon decays in pp collisions at $7 \mathrm{TeV}$, Phys. Rev. Lett. 107 (2011) 191802 [arXiv:1107.5834] [INSPIRE].

[37] Muon G-2 collaboration, G. Bennett et al., Measurement of the positive muon anomalous magnetic moment to 0.7 ppm, Phys. Rev. Lett. 89 (2002) 101804 [Erratum ibid. 89 (2002) 129903] [hep-ex/0208001] [INSPIRE].

[38] Muon G-2 collaboration, G. Bennett et al., Measurement of the negative muon anomalous magnetic moment to 0.7 ppm, Phys. Rev. Lett. 92 (2004) 161802 [hep-ex/0401008] [INSPIRE].

[39] Muon G-2 collaboration, G. Bennett et al., Final Report of the Muon E821 Anomalous Magnetic Moment Measurement at BNL, Phys. Rev. 73 (2006) 072003 [hep-ex/0602035] [INSPIRE].

[40] XENON1T collaboration, E. Aprile, The XENON1T Dark Matter Search Experiment,

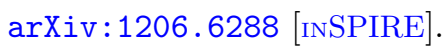

[41] SuPERCDMS collaboration, P.L. Brink, Conceptual Design for SuperCDMS SNOLAB, J. Low. Temp. Phys. 167 (2012) 1093 [InSPIRE].

[42] XENON100 collaboration, E. Aprile et al., Dark Matter Results from 225 Live Days of XENON100 Data, Phys. Rev. Lett. 109 (2012) 181301 [arXiv:1207.5988] [InSPIRE].

[43] Q. Shafi and C. Wetterich, Modification of GUT Predictions in the Presence of Spontaneous Compactification, Phys. Rev. Lett. 52 (1984) 875 [INSPIRE].

[44] C.T. Hill, Are There Significant Gravitational Corrections to the Unification Scale?, Phys. Lett. B 135 (1984) 47 [INSPIRE].

[45] X. Calmet, S.D. Hsu and D. Reeb, Grand unification and enhanced quantum gravitational effects, Phys. Rev. Lett. 101 (2008) 171802 [arXiv:0805.0145] [INSPIRE]. 
[46] J. Chakrabortty and A. Raychaudhuri, GUTs with dim-5 interactions: Gauge Unification and Intermediate Scales, Phys. Rev. D 81 (2010) 055004 [arXiv:0909.3905] [INSPIRE].

[47] LHCB collaboration, First Evidence for the Decay $B_{s}^{0} \rightarrow \mu^{+} \mu^{-}$, Phys. Rev. Lett. 110 (2013) 021801 [arXiv: 1211.2674$]$ [INSPIRE].

[48] BaBar collaboration, J. Lees et al., Evidence of $B \rightarrow \tau \nu$ decays with hadronic $B$ tags, Phys. Rev. D 88 (2013) 031102 [arXiv:1207.0698] [INSPIRE].

[49] Belle collaboration, I. Adachi et al., Measurement of $B^{-} \rightarrow \tau^{-} \bar{\nu}_{\tau}$ with a Hadronic Tagging Method Using the Full Data Sample of Belle, Phys. Rev. Lett. 110 (2013) 131801 [arXiv: 1208.4678] [INSPIRE].

[50] M. Davier, A. Hoecker, B. Malaescu and Z. Zhang, Reevaluation of the Hadronic Contributions to the Muon g-2 and to alpha(MZ), Eur. Phys. J. C 71 (2011) 1515 [Erratum ibid. C 72 (2012) 1874] [arXiv: 1010.4180] [INSPIRE].

[51] A. Djouadi, J.-L. Kneur and G. Moultaka, SuSpect: A Fortran code for the supersymmetric and Higgs particle spectrum in the MSSM, Comput. Phys. Commun. 176 (2007) 426 [hep-ph/0211331] [INSPIRE].

[52] G. Bélanger, F. Boudjema, A. Pukhov and A. Semenov, Dark matter direct detection rate in a generic model with MicrOMEGAs 2.2, Comput. Phys. Commun. 180 (2009) 747 [arXiv: 0803.2360] [INSPIRE].

[53] I. Gogoladze, F. Nasir and Q. Shafi, $\mathrm{SO}(10)$ as a Framework for Natural Supersymmetry, JHEP 11 (2013) 173 [arXiv: 1306.5699] [INSPIRE].

[54] J. Hisano, S. Matsumoto, O. Saito and M. Senami, Heavy wino-like neutralino dark matter annihilation into antiparticles, Phys. Rev. D 73 (2006) 055004 [hep-ph/0511118] [INSPIRE].

[55] S.P. Martin and J.D. Wells, Superconservative interpretation of muon g-2 results applied to supersymmetry, Phys. Rev. D 67 (2003) 015002 [hep-ph/0209309] [INSPIRE].

[56] D. Stöckinger, The Muon Magnetic Moment and Supersymmetry, J. Phys. G 34 (2007) R45 [hep-ph/0609168] [INSPIRE].

[57] G.-C. Cho, K. Hagiwara, Y. Matsumoto and D. Nomura, The MSSM confronts the precision electroweak data and the muon g-2, JHEP 11 (2011) 068 [arXiv:1104.1769] [INSPIRE].

[58] M. Endo, K. Hamaguchi, S. Iwamoto and T. Yoshinaga, Muon $g-2$ vs LHC in Supersymmetric Models, JHEP 01 (2014) 123 [arXiv:1303.4256] [INSPIRE].

[59] M. Adeel Ajaib, I. Gogoladze, Q. Shafi and C.S. Un, A Predictive Yukawa Unified SO(10) Model: Higgs and Sparticle Masses, JHEP 07 (2013) 139 [arXiv:1303.6964] [INSPIRE].

[60] S. Mohanty, S. Rao and D. Roy, Predictions of a Natural SUSY Dark Matter Model for Direct and Indirect Detection Experiments, JHEP 11 (2012) 175 [arXiv:1208.0894] [INSPIRE].

[61] S. Mohanty, S. Rao and D. Roy, Reconciling the muon $g-2$ and dark matter relic density with the LHC results in nonuniversal gaugino mass models, JHEP 09 (2013) 027 [arXiv: 1303.5830] [INSPIRE].

[62] S. Akula and P. Nath, Gluino-driven Radiative Breaking, Higgs Boson Mass, Muon $\mathbf{g}-\mathbf{2}$ and the Higgs Diphoton Decay in SUGRA Unification, Phys. Rev. D 87 (2013) 115022 [arXiv: 1304.5526] [INSPIRE]. 